\title{
BMJ Open Azithromycin as an add-on treatment for persistent uncontrolled asthma in adults: protocol of a systematic review and meta-analysis
}

\author{
Wei Liu, ${ }^{1}$ Wei Mu, ${ }^{2}$ Huiting Zhang, ${ }^{3}$ Jingbo Zhai, ${ }^{4}$ Xiaodan $L i,{ }^{5}$ Peng Guan, ${ }^{6}$ \\ Fu Lian, ${ }^{1}$ Jihong Feng, ${ }^{1}$ Shuangjiang Yu, ${ }^{3}$ Xuepin Wang, ${ }^{3}$ Jinhua $\mathrm{Si}^{7}$ \\ Zengtao Sun (D), ${ }^{8}$ Yuhong Huang ${ }^{2}$
}

To cite: Liu W, Mu W, Zhang $\mathrm{H}$, et al. Azithromycin as an addon treatment for persistent uncontrolled asthma in adults: protocol of a systematic review and meta-analysis. BMJ Open 2020;10:e032770. doi:10.1136/ bmjopen-2019-032770

- Prepublication history and additional material for this paper are available online. To view these files, please visit the journal online (http://dx.doi org/10.1136/bmjopen-2019032770).

WL and WM contributed equally.

Received 04 July 2019

Revised 04 December 2019

Accepted 05 December 2019

Check for updates

(c) Author(s) (or their employer(s)) 2020. Re-use permitted under CC BY-NC. No commercial re-use. See rights and permissions. Published by BMJ.

For numbered affiliations see end of article.

Correspondence to Dr Zengtao Sun; sunzengtaopro@163.com

Dr Yuhong Huang;

hyh101@126.com

\section{ABSTRACT}

Introduction Clinical management of asthma remains a public challenge. Despite standard treatment with inhaled corticosteroids (ICS) and long-acting beta-agonists (LABAs), asthma remains uncontrolled in a substantial number of chronic asthma patients who risk reduced lung function and severe exacerbations. Azithromycin could have add-on effects for these patients. This study is proposed to systematically evaluate the efficacy of azithromycin as an add-on treatment for adults with persistent uncontrolled symptomatic asthma.

Methods and analysis Two reviewers will perform a comprehensive search of PubMed, Embase, the Cochrane Central Register of Controlled Trials (CENTRAL) and four Chinese electronic databases including China National Knowledge Infrastructure (CNKI), Chinese Biomedical Literature Database (CBM), WanFang Data and VIP Database from inception to May 2019. Only randomised controlled trials will be included. There is no restriction on language or publication status. Combined oral azithromycin and an ICS or/and a LABA will be compared with standard treatment alone or with a placebo. The primary outcomes are the number or frequency of asthma exacerbations, changes in asthma symptoms and lung function. Secondary outcomes include the number or frequency of inhalations of beta-agonists with or without corticosteroids for rescue use, eosinophil counts in blood or sputum, adverse events and others. A meta-analysis will be attempted to provide an estimate of the pooled treatment effect. Otherwise, qualitative descriptions of individual studies will be given.

Ethics and dissemination Ethical approval is not required because no primary data will be collected. Study findings will be presented at scientific conferences or published in a peer-reviewed journal.

PROSPERO registration number CRD42019117272.

\section{INTRODUCTION}

Asthma is a major non-communicable chronic disease characterised by variable respiratory symptoms and airflow limitation. ${ }^{1}$ It cannot be cured, and currently affects around 334 million people globally. ${ }^{2}$ Undertreatment and failure in
Strengths and limitations of this study

- Randomised controlled clinical trials will be included to provide unbiased estimates of treatment effects.

- Recently published high-quality trials will be included to generate state-of-the-art and reliable evidence.

- Differences in the outcome measures reported may cause problems in data pooling.

treatment adherence may lead to disease exacerbations, lifetime disability and even death, creating huge burdens on individuals, families and public health. ${ }^{3}$ Studies estimate $10 \%-20 \%$ of patients experience persistent uncontrolled symptoms even on maximised maintenance therapy with inhaled corticosteroids (ICS) or/and long-acting beta-agonists (LABAs). ${ }^{4-6}$ Persistent uncontrolled asthma is characterised by three or more of the following signs: (1) daytime asthma symptoms more than two times per week; (2) night waking due to asthma; (3) reliever needed for symptoms more than two times per week and (4) any activity limitation due to asthma. ${ }^{7}$ These patients are at higher risk for asthma exacerbations, lifetime disability and even death. ${ }^{89}$ Prevention and control of asthma is high on the WHO's agenda. ${ }^{3}$ The current clinical management of uncontrolled asthma is not satisfactory and warrants additional treatment options.

The aetiology of asthma has not been understood well enough to provide clear guidance for clinical practice. Previous research has recognised three typical airway inflammatory phenotypes, that is, eosinophilic, non-eosinophilic and mixed granulocytic inflammation. ${ }^{1}$ Eosinophilic asthma correlates with the $\mathrm{Th}_{\mathrm{g}}$ /allergic pathways and responds well to steroid therapy. ${ }^{11}$ Non-eosinophilic asthma is possibly caused by bacterial or viral 
infections and particulate air pollution, thus being unresponsive to steroid therapy. ${ }^{12}$ There is evidence that a portion of asthma is based on non-eosinophilic airway inflammation or mixed granulocytic inflammation, ${ }^{13}$ which suggests antibiotics may have a role to play in asthma control.

Macrolides are a class of antibiotics that have been reported clinically effective for all phenotypes of asthma. ${ }^{11} 1415$ In addition to their direct antimicrobial activity, evidence indicates that macrolides also have antiinflammatory and immunomodulatory effects. ${ }^{16} 17$ Azithromycin is a second-generation macrolide antibiotic with anti-inflammatory and immunomodulatory functions and a good pharmacokinetic profile. ${ }^{18}$ Findings from clinical and review studies ${ }^{10} 1819$ suggest azithromycin as an add-on therapy may bring benefits for patients experiencing persistent uncontrolled asthma.

A 2012 randomised controlled trial (RCT) reports that compared with a placebo, $500 \mathrm{mg}$ of azithromycin three times weekly for 6 months reduced the rate of event-based asthma exacerbations by 0.98 per person and prolonged time to first exacerbation in patients with bronchiectasis. But, it did not improve lung function and health-related quality of life. ${ }^{19}$ A 2017 RCT involving 420 persistent asthma patients finds adding $500 \mathrm{mg}$ of azithromycin three times weekly to maintenance therapy for 48 weeks reduced asthma exacerbations by 0.79 per patient-year compared with a placebo and significantly improved asthma-related quality of life. ${ }^{10}$ A 2015 meta-analysis of 17 RCTs involving data from 1306 asthma patients suggests long-term (4 weeks and above) macrolides antibiotics therapy improved forced expiratory volume in one second $\left(\mathrm{FEV}_{1}\right.$, weighted mean difference: $0.11, \mathrm{p}<0.01$ ), peak expiratory flow (PEF, standard mean difference: $0.25, \mathrm{p}<0.001$ ), airway hyperresponsiveness, forced vital capacity (FVC) and $\mathrm{FEV}_{1} / \mathrm{FVC}$, but did not change other lung function indicators, clinical symptoms or quality of life. Seven of the 17 included studies use azithromycin. ${ }^{18}$

As far as we know, no previous systematic review has focused on the effect of azithromycin as an adjuvant to standard treatment for persistent uncontrolled asthma. Individual RCTs came to similar findings for some outcomes and conflicting findings for other outcomes. Moreover, the most relevant meta-analysis ${ }^{18}$ has evident heterogeneity as it examines macrolides as a whole, and it fails to include clinically meaningful outcomes such as exacerbation frequency.

With recent publications of high-quality RCTs on this subject, ${ }^{1011}{ }^{19-24}$ we aim to perform a systematic review and meta-analysis to investigate the effect of azithromycin as an add-on treatment for persistent uncontrolled asthma in adult patients.

\section{METHODS AND ANALYSIS}

This protocol is developed in accordance with the Preferred Reporting Items for Systematic Reviews and Meta-Analyses Protocols 2015 statement. ${ }^{25}$
Inclusion criteria

Types of study

Studies to be included should meet the following criteria: (1) parallel-group RCTs; (2) evaluate the efficacy and safety of azithromycin as an add-on therapy in addition to standard treatment with ICS or/and LABAs for persistent uncontrolled asthma in the adult population. There is no restriction on publication status or language.

\section{Types of participants}

The participants are adults with a definite diagnosis of uncontrolled asthma based on at least one of the current or past definitions or guidelines for asthma, such as the Global Initiative for Asthma Guideline. ${ }^{7}$

\section{Interventions and comparisons}

The experimental intervention should be oral azithromycin. The control intervention could be a placebo or no treatment. Trials will be excluded if other types of macrolide antibiotics are used. Standard treatment refers to ICS or/and LABAs. The treatment duration should be at least 4 weeks.

We will draw the following comparisons:

1. Azithromycin plus standard treatment compared with standard treatment alone.

2. Azithromycin plus standard treatment compared with placebo plus standard treatment.

Types of outcome measures

\section{Primary outcomes}

1. Number or frequency of asthma exacerbations. An exacerbation is defined as a deterioration of asthma symptoms requiring short-term systemic corticosteroid treatment, an asthma-related hospitalisation or an emergency room visit.

2. Changes in asthma symptoms. Measurement tools include, but are not limited to, the Asthma Control Test, ${ }^{26}$ the 5-item Asthma Control Questionnaire ${ }^{27}$ and the Asthma Quality of Life Questionnaire. ${ }^{28}$

3. Lung function indicators, including prebronchodilator $\mathrm{FEV}_{1}, \mathrm{FEV}_{1} \%$ of predicted value and PEF and so on.

\section{Secondary outcomes}

1. Number or frequency of inhalations of beta-agonists with or without corticosteroids for rescue use.

2. Eosinophil counts in blood or sputum.

3. Changes in fractional exhaled nitric oxide level from baseline.

4. Type and number of adverse events.

5. The number of participant drop-outs.

\section{Search strategy}

The review authors responsible for literature searching have been trained in hands-on workshops in this field. We carefully design our search strategy and have a librarian (JS) critically revise the searches to match each database. The searches will be updated before the review is ready for publication. 


\section{Electronic searches}

Two reviewers (WM and HZ) will perform a comprehensive search of PubMed, Embase, the Cochrane Central Register of Controlled Trials (CENTRAL) and four Chinese databases including China National Knowledge Infrastructure (CNKI), Chinese Biomedical Literature Database (CBM), WanFang and VIP databases from inception to May 2019 to identify potentially eligible studies. Bridging searches will also be conducted to capture literature published from June 2019 until the final review publication.

An example of our search strategy for PubMed using a combination of MeSH terms and free words is reported in online supplementary appendix 1 . There will be no restriction on the language and status of publication.

\section{Searching other resources}

We will review the reference lists of identified relevant articles for the possible inclusion of additional studies. Major international clinical trial registries such as ClinicalTrials.gov, the Chinese Clinical Trial Registry and the WHO International Clinical Trials Registry Platform will be searched to identify ongoing or unpublished trials. Google Scholar will be searched to identify grey literature further.

\section{Data collection and analysis}

Study selection

Records from electronic database searches will be imported into EndNote to eliminate duplications. Two review authors (WL and WM) will screen citations first by reviewing the title and abstract, and then by reading the full-text according to the prespecified inclusion and exclusion criteria. The decision for including a study will be made independently by each reviewer. Any disagreement will be resolved by discussion or further consultation with a third reviewer (HZ).

\section{Data extraction and management}

Two review authors (WL and WM) will independently extract and record the following data from each included study:

1. Publication details: title, authors, publication year, funding and country.

2. Study details: aim, design, inclusion and exclusion criteria, randomisation method, use of allocation concealment, blinding and description of follow-up.

3. Patient characteristics: age, gender, sample size, the number of patients included in the analysis, phenotype of asthma, patient compliance and disease course.

4. Intervention information: duration, dose and dosage, and standard treatment.

5. Outcome: outcome measures reported, tools for outcome assessment and adverse events.

Assessment of risk of bias in included studies

The risks of bias for an individual study will be assessed by two authors (WL and WM) based on the approach recommended by the Cochrane Handbook for Systematic
Reviews of Interventions. ${ }^{29}$ The following domains will be examined: random sequence generation, blinding of participants and personnel, blinding of outcome assessment, incomplete outcome data, selective outcome reporting and other bias. We will grade each source of bias as high, low or unclear. The ratings and corresponding clarifications will be presented in the 'Risk of Bias Table' with a quote from the study report. A study will be considered at low risk of bias if randomisation sequence generation, blinding and outcome data reporting are adequately performed and reported. ${ }^{29}$

\section{Measures of treatment effect}

The risk ratio with $95 \%$ CI will be used to assess the estimated pooled effect for dichotomous outcomes, and the mean difference with 95\% CI will be used for continuous outcomes. Moreover, the standard mean difference with 95\% CI will be used to present an intervention effect if the same outcome is measured in various ways.

\section{Assessment of heterogeneity}

The $\chi^{2}$ test and $\mathrm{I}^{2}$ will be used to assess heterogeneity for the study outcomes. An $\mathrm{I}^{2}$ value $>50 \%$ or a $\mathrm{p}$ value $<0.10$ indicates the presence of significant statistical heterogeneity. Subgroup analyses and sensitivity analyses will also be performed to assess potential heterogeneity.

\section{Assessment of reporting biases}

If it is possible to include 10 or more studies for one common outcome, we will create a funnel plot to examine publication bias. ${ }^{30}$

\section{Data synthesis}

Data will be synthesised to provide estimates of the pooled intervention effect using meta-analysis if these trials evaluate the same intervention for a common outcome with comparable methods. The inverse variance method will be used for continuous data and the Mantel-Haenszel method for dichotomous data. ${ }^{31}$

The fixed-effect model will be used for data pooling if statistical heterogeneity is low $\left(\mathrm{p}>0.1\right.$ or $\left.\mathrm{I}^{2}<50 \%\right)$. Otherwise, the random-effect model will be adopted to provide a more reasonable estimate of effect. ${ }^{29}$ However, if one or two studies dominate the evidence, the random-effect model may grossly overestimate the effect size. ${ }^{32}$ In that case, the fixed-effect model will be used to make a more conservative conclusion. The Review Manager V.5.3 software will be used. If sparse data are collected for an outcome which does not allow for quantitative analysis, a narrative summary of the findings from individual studies will be provided instead.

\section{Subgroup analysis and investigation of heterogeneity}

We will develop the following subgroup analyses to assess possible clinical heterogeneity (if sufficient data are available) according to:

1. Inflammatory phenotypes (eg, eosinophilic vs noneosinophilic asthma subtype based on serological test results). 
2. Treatment durations (eg, $<6$ vs 6 months and above).

3. Azithromycin dosage or treatment regimens.

4. Patient age groups (eg, categorising trials into 18-30, $30-45$ and above 45 age groups according to mean age).

5 . The comparator intervention (eg, a blank vs a placebo). The $\chi^{2}$ test will be used in analysing the intervention effect. A p value of $<0.05$ indicates a statistically significant difference between subgroups.

\section{Sensitivity analysis}

We will perform a sensitivity analysis to assess the reliability of the pooled results by excluding trials with a high risk of bias or trials reporting data missing.

Summary of findings table and quality of evidence

The web-based GRADEpro GDT ${ }^{33}$ will be adopted to create a summary of findings table for important outcomes. Quality of the evidence will be evaluated by two reviewers (WL and WM) from five aspects: study limitations, inconsistency, imprecision, indirectness and publication bias. Gradings for evidence quality could be high, moderate, low or very low. Any disagreement will be resolved by discussion with a third review author (HZ).

\section{Patient and public involvement}

We did not seek patient or public involvement in the draft of the protocol. Nor will we enlist patient and public opinion or help in the conduct of this systematic review and meta-analysis.

\section{Amendments}

The date, the rationale and a description of the changes made will be provided in the event of protocol amendments.

\section{ETHICS AND DISSEMINATION}

Ethical approval is not required because no primary data are collected. This review will provide a comprehensive assessment of the efficacy of azithromycin as an add-on treatment for persistent uncontrolled asthma in adults. Findings of this study could be used as a reference for the clinical management of the target population. Findings of this meta-analysis will be presented at scientific conferences and be published in scientific journals.

\section{Protocol registration and progress}

This review protocol is registered with PROSPERO. As of October 2019, the reviewers have completed the literature search and are screening trials.

\section{Author affiliations}

${ }^{1}$ Department of Respiratory Medicine, Second Affiliated Hospital of Tianjin University of Traditional Chinese Medicine, Tianjin, China

${ }^{2}$ Department of Clinical Pharmacology, Second Affiliated Hospital of Tianjin University of Traditional Chinese Medicine, Tianjin, China

${ }^{3}$ Graduate School, Tianjin University of Traditional Chinese Medicine, Tianjin, China ${ }^{4}$ Institute of Traditional Chinese Medicine, Tianjin University of Traditional Chinese Medicine, Tianjin, China
${ }^{5} \mathrm{VIP}$ Inpatient Ward, Second Affiliated Hospital of Tianjin University of Traditional Chinese Medicine, Tianjin, China

${ }^{6}$ Department of Emergency Medicine, Second Affiliated Hospital of Tianjin University of Traditional Chinese Medicine, Tianjin, China

${ }^{7}$ Library, Tianjin University of Traditional Chinese Medicine, Tianjin, China

${ }^{8}$ Office of Hospital Management, Tianjin University of Traditional Chinese Medicine, Tianjin, China

Contributors WL and WM conceived the study and designed the protocol. WL, WM, $\mathrm{HZ}$ and JZ drafted the manuscript. WM and $\mathrm{HZ}$ developed the search strategy. JS revised the search strategy and adapted searches across different electronic databases. XL, PG, FL, JF, SY and XW provided general guidance to the development of the protocol and critically revised the manuscript. ZS and $\mathrm{YH}$ reviewed and revised the manuscript for important intellectual content. All authors read and approved the final manuscript.

Funding This work was supported by the National Natural Science Foundation of China grant numbers 81603447 and 81603495.

Disclaimer The funding agency plays no role in protocol development or in the decision to publish.

Competing interests None declared.

Patient consent for publication Not required.

Provenance and peer review Not commissioned; externally peer reviewed.

Open access This is an open access article distributed in accordance with the Creative Commons Attribution Non Commercial (CC BY-NC 4.0) license, which permits others to distribute, remix, adapt, build upon this work non-commercially, and license their derivative works on different terms, provided the original work is properly cited, appropriate credit is given, any changes made indicated, and the use is non-commercial. See: http://creativecommons.org/licenses/by-nc/4.0/.

ORCID iD

Zengtao Sun http://orcid.org/0000-0002-2505-8693

\section{REFERENCES}

1 Papi A, Brightling C, Pedersen SE, et al. Asthma. The Lancet 2018;391:783-800.

2 Vos T, Flaxman AD, Naghavi M, et al. Years lived with disability (YLDs) for 1160 sequelae of 289 diseases and injuries 1990-2010: a systematic analysis for the global burden of disease study 2010 . The Lancet 2012;380:2163-96.

3 World Health Organization. Asthma. Available: http://www.who.int/ en/news-room/fact-sheets/detail/asthma [Accessed 10 May 2019].

4 Bateman ED, Boushey HA, Bousquet J, et al. Can guideline-defined asthma control be achieved? the gaining optimal asthma control study. Am J Respir Crit Care Med 2004;170:836-44.

5 Hermosa JLR, Sánchez CB, Rubio MC, et al. Factors associated with the control of severe asthma. J Asthma 2010;47:124-30.

6 Peters SP, Ferguson G, Deniz Y, et al. Uncontrolled asthma: a review of the prevalence, disease burden and options for treatment. Respir Med 2006;100:1139-51.

7 The Global Initiative for Asthma Group. Pocket Guide for Asthma Management and Prevention. 2018; 9.. Available: https://ginasthma. org/wp-content/uploads/2018/03/wms- GINA-main-pocket-guide_ 2018-v1.0.pdf

8 Calhoun WJ, Haselkorn T, Mink DR, et al. Clinical burden and predictors of asthma exacerbations in patients on Guideline-based steps 4-6 asthma therapy in the TENOR cohort. J Allergy Clin Immunol 2014;2:193-200.

9 Ivanova JI, Bergman R, Birnbaum HG, et al. Effect of asthma exacerbations on health care costs among asthmatic patients with moderate and severe persistent asthma. J Allergy Clin Immunol 2012;129:1229-35.

10 Gibson PG, Yang IA, Upham JW, et al. Effect of azithromycin on asthma exacerbations and quality of life in adults with persistent uncontrolled asthma (AMAZES): a randomised, double-blind, placebo-controlled trial. The Lancet 2017;390:659-68.

11 Brusselle GG, Vanderstichele C, Jordens P, et al. Azithromycin for prevention of exacerbations in severe asthma (AZISAST): a multicentre randomised double-blind placebo-controlled trial. Thorax 2013;68:322-9.

12 Douwes Jet al. Non-Eosinophilic asthma: importance and possible mechanisms. Thorax 2002;57:643-8. 
13 Haldar P, Pavord ID. Noneosinophilic asthma: a distinct clinical and pathologic phenotype. J Allergy Clin Immunol 2007;119:1043-52.

14 Altenburg J, de Graaff CS, van der Werf TS, et al. Immunomodulatory effects of macrolide antibiotics - Part 2: advantages and disadvantages of long-term, low-dose macrolide therapy. Respiration 2011;81:75-87.

15 Simpson JL, Powell H, Boyle MJ, et al. Clarithromycin targets neutrophilic airway inflammation in refractory asthma. Am J Respir Crit Care Med 2008;177:148-55.

16 Parnham MJ. Immunomodulatory effects of antimicrobials in the therapy of respiratory tract infections. Curr Opin Infect Dis 2005;18:125-31.

17 Tamaoki J, Kadota J, Takizawa H. Clinical implications of the immunomodulatory effects of macrolides. Am J Med 2004;117:5-11.

18 Tong X, Guo T, Liu S, et al. Macrolide antibiotics for treatment of asthma in adults: a meta-analysis of 18 randomized controlled clinical studies. Pulm Pharmacol Ther 2015;31:99-108.

19 Wong C, Jayaram L, Karalus N, et al. Azithromycin for prevention of exacerbations in non-cystic fibrosis bronchiectasis (embrace): a randomised, double-blind, placebo-controlled trial. The Lancet 2012;380:660-7.

20 Johnston SL, Szigeti M, Cross M, et al. Azithromycin for acute exacerbations of asthma : The AZALEA randomized clinical trial. JAMA Intern Med 2016;176:1630-7.

21 Hahn DL, Grasmick M, Hetzel S, et al. Azithromycin for bronchial asthma in adults: an effectiveness trial. J Am Board Fam Med 2012;25:442-59.

22 Cameron EJ, Chaudhuri R, Mair F, et al. Randomised controlled trial of azithromycin in smokers with asthma: table 1-. Eur Respir J 2013;42:1412-5.

23 Busse WW, Maspero JF, Rabe KF, et al. Liberty asthma quest: phase 3 randomized, double-blind, placebo-controlled, parallelgroup study to evaluate dupilumab efficacy/safety in patients with uncontrolled, moderate-to-severe asthma. Adv Ther 2018;35:737-48.

24 Castro M, Corren J, Pavord ID, et al. Dupilumab efficacy and safety in moderate-to-severe uncontrolled asthma. N Engl J Med 2018;378:2486-96.

25 Shamseer L, Moher D, Clarke M, et al. Preferred reporting items for systematic review and meta-analysis protocols (PRISMA-P) 2015 : elaboration and explanation. BMJ 2015;349:g7647.

26 Nathan RA, Sorkness CA, Kosinski M, et al. Development of the asthma control test ¿ $^{\mathrm{A}}$ s survey for assessing asthma control. J Allergy Clin Immunol 2004;113:59-65.

27 Juniper EF, Svensson K, Mörk A-C, et al. Measurement properties and interpretation of three shortened versions of the asthma control questionnaire. Respir Med 2005;99:553-8.

28 Juniper EF, Buist AS, Cox FM, et al. Validation of a standardized version of the asthma quality of life questionnaire. Chest 1999;115:1265-70.

29 Higgins JPT, Green S. Cochrane Handbook for systematic reviews of interventions version 5.1.0. 2011: the Cochrane collaboration. Available: http://www.handbook.cochrane.org [Accessed Mar 2011]

30 Chauhan BF, Chong J, Asher I. Antileukotriene agents compared with placebo in children and adults with mild asthma (protocol). Cochrane Database Syst Rev 2015;9.

31 Edwards M, Gallagher A, Nair P, et al. Anti-interleukin-13 and anti-interleukin-4 agents versus placebo, anti-interleukin-5 or anti-immunoglobulin-E agents, for children and adults with asthma. Cochrane Database Syst Rev 2018;112.

32 Barbateskovic M, Schjørring OL, Jakobsen JC, et al. Higher versus lower inspiratory oxygen fraction or targets of arterial oxygenation for adult intensive care patients (protocol). Cochrane Database Syst Rev 2017; 4

33 GRADEpro GDT. GRADEpro Guideline development tool, 2015. Available: http://gdt.guidelinedevelopment.org/ 